\title{
Prevalence of Heterophoria and Associations with Refractive Error, Heterotropia and Ethnicity in Australian School Children
}

Jody Fay Leone, Annette Kifley, lan G Morgan, et al.

Br J Ophthalmo/ published online October 22, 2009

doi: 10.1136/bjo.2009.163709

Updated information and services can be found at:

http://bjo.bmj.com/content/early/2009/10/21/bjo.2009.163709

These include:

$\mathbf{P}<\mathbf{P} \quad$ Published online October 22, 2009 in advance of the print journal.

Email alerting Receive free email alerts when new articles cite this article. Sign up in the box at service the top right corner of the online article.

\section{Notes}

Advance online articles have been peer reviewed and accepted for publication but have not yet appeared in the paper journal (edited, typeset versions may be posted when available prior to final publication). Advance online articles are citable and establish publication priority; they are indexed by PubMed from initial publication. Citations to Advance online articles must include the digital object identifier (DOIs) and date of initial publication. 


\title{
Prevalence of Heterophoria and Associations with Refractive Error, Heterotropia and Ethnicity in Australian School Children
}

\author{
Jody F. Leone ${ }^{1}$ \\ Elaine Cornell ${ }^{1}$ \\ Ian G. Morgan ${ }^{2}$ \\ Paul Mitchell ${ }^{3}$ \\ Annette Kifley $^{3}$ \\ Jie Jin Wang ${ }^{3}$ \\ Kathryn A. Rose ${ }^{1}$
}

1. Discipline of Orthoptics, Faculty of Health Sciences, University of Sydney, Australia.

2. ARC Centre of Excellence in Vision Science, School of Biology, The Australian

National University, Canberra, Australia.

3. Department of Ophthalmology (Centre for Vision Research, Westmead Millennium Institute, Westmead Hospital), University of Sydney, Westmead, Australia.

Corresponding author and reprint requests:

Dr Kathryn Rose

Discipline of Orthoptics, Faculty of Health Sciences, University of Sydney

(Cumberland Campus),

PO Box 170,

Lidcombe NSW 1825, Australia.

Email: k.rose@usyd.edu.au

Word Count: 2607

The Corresponding Author has the right to grant on behalf of all authors and does grant on behalf of all authors, an exclusive licence (or non exclusive for government employees) on a worldwide basis to the BMJ Publishing Group Ltd and its Licensees to permit this article to be published in BJO editions and any other BMJPGL products to exploit

All subsidiary rights, as set out in our licence

(http://group.bmj.com/products/journals/instructions-for-authors/licence-forms). 


\section{ABSTRACT}

Aims: To establish the prevalence of heterophoria and its association with refractive error and ethnicity in a population-based study of Australian school children

Methods: The Sydney Myopia Study is a stratified, random cluster (school-based) sample of 4093 students (examined: 2003-2005). Two samples aged $6(n=1692)$ and 12 years $(n=2289)$ without heterotropia were included. Prevalent heterophoria was assessed using cover un-cover and prism bar alternate cover testing at $33 \mathrm{~cm}$ and $6 \mathrm{~m}$ distance fixation. Cycloplegic autorefraction (1\% cyclopentolate) was performed. Significant refractive error was defined as $\leq-$ $0.50 \mathrm{SE}$ and $\geq+2.00 \mathrm{SE}$.

Results: Exophoria was highly prevalent at near fixation (age 6: 58.3\%, age 12: 52.2\%). Orthophoria predominated at distance fixation (age 6: 85.4\%, age 12: 90.9\%). Hyperopia was associated with esophoria at near (age 6: odds ratio [OR] 1.7, 95\% confidence interval (CI) 1.1-2.8, age 12: OR 2.9, CI 1.1-2.8) and distance fixation (age 6: OR 9.7, CI 3.5-26, age 12: 9.6 OR, CI 4.2-22). Myopia was associated with exophoria at near (OR 2.1, CI 1.5-2.7) and distance fixation (OR 3.1, CI 2.1-4.4) for 12 year old children only. Exophoria was more frequent in children of East Asian than European Caucasian origins, even after adjusting for refraction; at near (age 6: OR 1.4, CI 1.0-2.0, age 12: OR 1.4, CI 1.0-1.9) and distance (age 12: OR 1.7, CI 1.1-2.7).

Conclusion: Contrary to other studies, exophoria, not orthophoria, was predominant for near. Exophoria was more prevalent in children of East Asian origin. Longitudinal studies are needed to establish if incident heterotropia is preceded by heterophoria.

Word Count Abstract only: 250

Key Words: Strabismus/Phoria, Exotropia/Exophoria/Esotropia/Esophoria, Refractive Errors, Ethnic Groups, Epidemiology 
Heterophoria is a latent deviation of the eyes that is only revealed when fusional vergence is disrupted, allowing the eyes to assume their position of rest. ${ }^{1}$ Upon resumption of binocular viewing, fusional vergence is used to return the eyes to alignment. Heterophoria is categorised by the direction of movement of the eyes when they are dissociated; esophoria describes an inward horizontal movement, while exophoria is an outward movement. Orthophoria is the absence of any movement on dissociation. Dissociative vertical movements of the eyes, either upwards (hyperphoria) or downwards (hypophoria) are infrequent, but can sometimes occur in combination with horizontal heterophoria's.

While heterophoria is generally without symptoms, poorly controlled heterophoria may lead to symptoms such as headache, blurred vision, diplopia or confusion, with the possibility of a manifest intermittent heterotropia. ${ }^{1}$ Failure to compensate for a heterophoria will result in a heterotropia, but the failure of compensation does not depend on the size of the heterophoria, but rather on inadequate fusional reserves.

There have been differences in the reported prevalence ${ }^{2-8}$ and distribution of heterophoria at near. ${ }^{349}$ However, previous studies of children are in agreement that orthophoria predominates at distance fixation, ${ }^{235}$ with a relatively tight (leptokurtic) distribution. ${ }^{34910}$ Heterophoria prevalence has been reported for European Caucasian populations, ${ }^{2-5}$ but there are no reports for East Asian samples. Factors reported to be associated with heterophoria include age ${ }^{237}$ and gender. ${ }^{11}$ While a relationship between heterophoria and refractive error has often been claimed, ${ }^{12}{ }^{13}$ studies specifically investigating this link have reported no association, ${ }^{4}$ or no direct relationship between the amount of ametropia and heterophoria. ${ }^{11}$ In this paper, we aim to document the prevalence of heterophoria and its subtypes in a large school-based sample of Australian school-aged children and to examine its associations.

\section{MATERIALS AND METHODS}

The Sydney Myopia Study was a school-based, cross sectional, survey of refraction and eye health in 4093 Sydney schoolchildren during 2003-2005. Detailed study methods have been described elsewhere. ${ }^{14}$ Schools were randomly selected using a stratified cluster sampling design.

All tests of ocular alignment were performed by orthoptists. Corneal reflections (Hirschberg test) were observed, followed by a cover/uncover test. Children with manifest heterotropia were excluded from analysis. In all other children, a slow alternate cover test (using a Clement Clarke Ltd. occluder, Haag-Streit, UK) was performed to fully dissociate the eyes and reveal any heterophoria. At $33 \mathrm{~cm}$, the Clement Clark fixation stick, with standardised detailed targets was used. Initially the target was briefly moved sideways to ensure uniocular fixation. The child was also asked to describe detail within the target, older children read the reduced Snellens optotypes. At $6 \mathrm{~m}$, a detailed picture was used. If a child wore glasses, both the prevalent heterophoria (without glasses) and the presenting heterophoria (with habitual glasses worn) were assessed. Heterophoria size was measured using the alternate prism cover test ${ }^{15}$ using Luneau prism bars in 2 prism dioptre (PD) steps, and classified as $\geq 2 \mathrm{PD}$ of esophoria, exophoria, or hyperphoria. Orthophoria was defined as a heterophoria between >2PD and <2PD. In this study, prevalent heterophoria was analysed unless specified otherwise. Heterophoria assessment was not performed using best corrected glasses, as this would induce a short-term change in heterophoria. ${ }^{16}$ 
Cycloplegic autorefraction was performed on all children (RK-F1 Auto Ref-Keratometer; Canon, Tokyo, Japan) using amethocaine (0.5\%) instilled 2 minutes prior to 1 drop each of cyclopentolate $(1 \%)$ and tropicamide $(1 \%)$ and repeated after 5 minutes. Autorefraction was performed an average 25 minutes after the instillation of the last drops. Cycloplegia was assessed by pupil diameter $\geq 6 \mathrm{~mm}$ and no reaction to light or accommodative target.

Clinically significant refractive error was defined for myopia as $\leq-0.50 \mathrm{SE}$ and for hyperopia if spherical equivalent refraction was $\geq+2.00 \mathrm{SE}$.

Data were entered into a Microsoft Access database (Microsoft, Redmond, WA). All statistical analysis was performed using Statistical Analysis System software, version 8.2 (SAS Institute, Cary, NC). The proportion of children with orthophoria, exophoria and esophoria was adjusted for cluster by schools. These rates are compared between age samples and demographic subgroups using chi-square tests for independence. Among children with heterophoria, mean PD of heterophoria were compared between age samples using twosample t-tests. Polytomous logistic regression with a generalised logit link was used to compare the odds of having heterophoria between children in different refractive and demographic subgroups. All odds ratios are unadjusted unless otherwise stated. In these analyses, orthophoria was used as the reference response category.

Approval for the study was obtained from the Human Research Ethics Committee, University of Sydney; the Department of Education and Training in New South Wales; Catholic Education Office and private schools. The study adhered to the tenets of the declaration of Helsinki. Informed written consent from at least one parent and verbal assent from each child were obtained prior to examination.

\section{RESULTS}

Two age cohorts, 1740 students in Year 1 (mean age 6.7 years, age range 5.5 to 8.1 years, standard deviation [SD] 0.42 years) and 2353 students in Year 7 (mean age 12.7 years, age range 11.1 to 14.4 years, SD 0.44 years) were examined, with response rates among eligible 6- and 12-year old children of $77.6 \%$ and $75.3 \%$, respectively. Among the children included in this study, $50.7 \%$ of the 6-year old children were boys, as were $50.8 \%$ of the 12 -year old children. Most children were of European Caucasian (63.4\% of the 6- and $60.0 \%$ of the 12 year old children) or East Asian ethnicity (17.3\% of the 6- and $14.9 \%$ of the 12 -year old children). Other ethnic groups were too small to permit meaningful analysis. Of 1740 children aged 6 years, we excluded 48 (2.8\%) children with heterotropia of whom 26 had esotropia, 14 exotropia, 7 microtropia and 1 child had VIth cranial nerve palsy. ${ }^{17}$ Of 2353 children aged 12 years, we excluded a similar proportion children with heterotropia $(2.7 \% \mathrm{n}=64)$, of whom 21 had esotropia, 27 exotropia, and 16 had a microtropia. ${ }^{18}$

\section{Prevalence of Heterophoria}

Figure 1 shows prevalence of heterophoria for the two age groups for near and distance fixation. At near fixation, exophoria was the most frequently occurring prevalent heterophoria (that is without glasses), present in $58.3 \%$ of the 6-year old and $52.2 \%$ of the 12 -year old children. At distance fixation, $85.4 \%$ of the 6 -year old and $90.9 \%$ of the 12 -year old children were orthophoric. Esophoria was rare at distance fixation $(1.0 \%, 1.3 \%$ for ages 6 and 12 respectively) and had a low prevalence at near fixation $(9.2 \%, 10.4 \%$ for ages 6 and 12 respectively), see Figure 1. There was a trend towards an increasing proportion of orthophoria 
in the older age group, and significantly less exophoria in the older age group (7.8\%) compared with the younger age group (13.5\%), at distance fixation $(p=0.007)$ (Figure 1). There was no significant difference found between the age groups in the other forms of heterophoria at either distance. Vertical heterophoria was detected in a small number of children at both near $(n=9,0.2 \%)$ and distance $(n=4,0.1 \%)$, mostly co-existing with a horizontal heterophoria. There were no significant gender differences in heterophoria, apart from 6-year old girls having more exophoria at near fixation (OR 1.4, 95\% CI 1.1-1.7).

\section{Size of Prevalent Heterophoria}

Figure 2 shows the distribution of heterophoria for the two age groups for near and distance fixation. A Kolmogorov-Smirnov goodness-of-fit test showed that the size (measured in PD) of horizontal prevalent heterophoria at both distances and in both age groups was not normally distributed $(p<0.0001)$. The distribution of near heterophoria for both age groups was mildly leptokurtic and skewed towards exophoria (kurtosis: 1.6 in children aged 6 years and 5.3 in children aged 12 years). Distance heterophoria was markedly leptokurtic and mildly skewed towards exophoria for both age groups (kurtosis: 20.2 in children aged 6 years and 38.5 in children aged 12 years) (Figure 2).

The average magnitude of prevalent heterophoria at near fixation was lower in the 12-year old (exophoria: 3.9PD, esophoria: 3.8PD) than in the 6-year old children (exophoria: 4.9PD $p<0.0001$, esophoria: 4.4PD $p=0.01$ ). However, the difference was not clinically significant, at only around 1PD. There was no significant age difference in prevalent heterophoria size noted for distance fixation.

\section{Large Prevalent Heterophoria}

A small number of children, $82(4.9 \%)$ aged 6 and $74(3.2 \%)$ aged 12, had a large prevalent heterophoria (that is $\geq 10 \mathrm{PD}$, maximum $25 \mathrm{PD})$, usually at near $(80.7 \%$ of cases aged 6 and $85.1 \%$ aged 12 years). The remainder were either at distance only (8.4\% of cases aged 6 and $6.8 \%$ aged 12 year) or at both near and distance fixation (10.8\% of cases aged 6 and $8.1 \%$ aged 12 years). In children with large heterophoria aged $12,45.6 \%$ had a significant refractive error in comparison to $17.4 \%$ of those without large heterophoria $(p<0.0001)$. However, in children with large heterophoria aged 6 , the proportion with refractive error was not significantly different (19\% with large heterophoria, $13.4 \%$ without, $p=0.16$ ).

\section{Relationship between Prevalent Heterophoria, Refractive Error and Ethnicity}

The proportion of children aged 6 with hyperopia was $12.1 \%$, and myopia $1.4 \%$. In the children aged 12 the proportion with hyperopia was lower (4.3\%) but myopia was higher $(12.3 \%)$. Table 1 shows the association between heterophoria and refractive error for the two age groups. Significant associations were found between cycloplegic refractive error and prevalent heterophoria (measured without glasses), for both near ( 6 year-old children: $p=0.03$, 12 year-old children: $p<0.0001$ ) and distance fixation (both age groups: $p<0.0001$ ). While esophoria was rare, children with hyperopia were significantly more likely than those without refractive error to have esophoria at near (age 6: OR 1.7; age 12: OR 2.9) and distance (age 6: OR 9.7; age 12: OR 9.6) (Table 1). The proportion of children with myopia and esophoria at near was less than $1 \%$ of the total sample, and $8.6 \%$ of the myopic population. In the older children, those with myopia were significantly more likely than those without refractive error 
to be exophoric at near and distance (Table 1). This association was not evident in the younger children, in whom very few had myopia.

Table 2 shows the prevalence between ethnicity and heterophoria for the two age groups. East Asian children were significantly more likely than European Caucasian children to be exophoric at near, in both 6 year (OR 1.4, 95\% CI 1.1-1.9) and 12 year groups (OR 1.8, 95\% CI 1.5-2.5), and at distance in the 12 year group (OR 2.7, 95\% CI 1.9-4.1) (Table 2). After adjusting for refractive error, gender and age, these ethnicity associations with exophoria at near, remained significant for both children aged 6 years (adjusted OR 1.4, 95\% CI 1.0-2.0) and 12 years (adjusted OR 1.4, 95\% CI 1.0-1.9), and the association with exophoria at distance remained significant for the 12 year-old group (adjusted OR 1.7, 95\% CI 1.1-2.7). Average inter-pupillary distance (IPD) in European Caucasian and East Asian children was not significantly different at age 6 years $(56.2 \mathrm{~mm}, 56 \mathrm{~mm}$ respectively, $p=0.4)$. While the average IPD in children aged 12 years is larger, there was still no significant difference between European Caucasian $(59.7 \mathrm{~mm})$ and East Asian children $(60.1 \mathrm{~mm})(p=0.7)$. 
Table 1 Number, proportion and odds ratios for prevalent heterophoria (without glasses) among children aged 6 and 12 years with different refractive status by myopia and hyperopia.

\begin{tabular}{|c|c|c|c|c|c|c|c|c|c|c|}
\hline & \multicolumn{5}{|c|}{ At Near } & \multicolumn{5}{|c|}{ At Distance } \\
\hline & \multirow{2}{*}{$\begin{array}{c}\begin{array}{c}\text { Orthophoria } \\
\text { reference } \\
\text { group }\end{array} \\
\mathrm{n}(\%)\end{array}$} & \multicolumn{2}{|c|}{ Esophoria } & \multicolumn{2}{|c|}{ Exophoria } & \multirow{2}{*}{$\begin{array}{c}\begin{array}{c}\text { Orthophoria } \\
\text { reference } \\
\text { group }\end{array} \\
\mathrm{n}(\%)\end{array}$} & \multicolumn{2}{|c|}{ Esophoria } & \multicolumn{2}{|c|}{ Exophoria } \\
\hline & & $\mathrm{n}(\%)$ & $\begin{array}{c}\mathrm{OR} \\
(95 \% \mathrm{CI})\end{array}$ & $\mathrm{n}(\%)$ & $\begin{array}{c}\mathrm{OR} \\
(95 \% \mathrm{CI})\end{array}$ & & $\mathrm{n}(\%)$ & $\begin{array}{c}\mathrm{OR} \\
(95 \% \mathrm{CI})\end{array}$ & $\mathrm{n}(\%)$ & $\begin{array}{c}\mathrm{OR} \\
(95 \% \mathrm{CI})\end{array}$ \\
\hline \multicolumn{11}{|l|}{ Age 6} \\
\hline $\begin{array}{l}\text { No significant } \\
\text { refractive error }\end{array}$ & $474(33)$ & $122(8)$ & 1.0 & $852(59)$ & 1.0 & $1224(86)$ & $7(0.5)$ & 1.0 & $\begin{array}{c}192 \\
(13.5)\end{array}$ & 1.0 \\
\hline Myopia ${ }^{\ddagger}$ & $5(21)$ & $1(4)$ & $0.8(0.1-6.7)$ & $18(75)$ & $2.0(0.7-5.4)$ & $20(91)$ & $0(-)$ & - & $2(9)$ & - \\
\hline \multirow[t]{2}{*}{ Hyperopia $^{\S}$} & $66(33)$ & $29(14)$ & $1.7(1.1-2.8)^{\mathbf{a}}$ & $107(53)$ & $0.9(0.6-1.3)$ & $163(82)$ & $9(5)$ & $9.7(3.5-26)^{\mathrm{a}}$ & $26(13)$ & $1.0(0.6-1.6)$ \\
\hline & \multicolumn{5}{|c|}{ Overall $p$ for association $=0.03$} & \multicolumn{5}{|c|}{ Overall p for association $<0.0001$} \\
\hline \multicolumn{11}{|l|}{ Age 12} \\
\hline $\begin{array}{l}\text { No significant } \\
\text { refractive error }\end{array}$ & $742(39)$ & $195(10)$ & 1.0 & $961(51)$ & 1.0 & $1743(93)$ & $20(1)$ & 1.0 & $120(6)$ & 1.0 \\
\hline Myopia & $72(26)$ & $13(5)$ & $0.7(0.4-1.3)$ & $192(69)$ & $2.1(1.5-2.7)^{\mathbf{b}}$ & $228(83)$ & $0(-)$ & - & $48(17)$ & $3.1(2.1-4.4)^{\mathbf{b}}$ \\
\hline Hyperopia $^{\S}$ & $36(37)$ & $27(28)$ & $2.9(1.7-4.8)^{\mathbf{a}}$ & $34(35)$ & $0.7(0.4-1.2)$ & $82(84)$ & $9(9)$ & $9.6(4.2-22)^{\mathbf{a}}$ & $7(7)$ & $1.2(0.6-2.7)$ \\
\hline & \multicolumn{5}{|c|}{ Overall $p$ for association $<0.0001$} & \multicolumn{5}{|c|}{ Overall $p$ for association $<0.0001$} \\
\hline
\end{tabular}

\footnotetext{
${ }^{\dagger}$ No significant refractive error defined as spherical equivalent $>-0.5 \mathrm{SE}$ to $<+2.00 \mathrm{SE}$

Myopia defined as: spherical equivalent $\leq-0.5 \mathrm{SE}$

${ }^{\S}$ Hyperopia defined as: spherical equivalent $\geq+2.00 \mathrm{SE}$

${ }^{a}$ The significant odds of esophoria rather than orthophoria was increased in those with hyperopia compared to those with no significant refractive error.

b The significant odds of exophoria rather than orthophoria was increased in those with myopia compared to those with no significant refractive error.
} 
Table 2 Prevalent heterophoria (without glasses) prevalence in children aged 6 and 12 years attending the Sydney Myopia Study, by ethnicity.

\begin{tabular}{|c|c|c|c|c|c|c|c|c|}
\hline & \multicolumn{3}{|c|}{ Esophoria } & \multicolumn{3}{|c|}{ Exophoria } & \multicolumn{2}{|r|}{$\begin{array}{l}\text { Orthophoria } \\
\text { reference group }\end{array}$} \\
\hline & $\mathrm{n}$ & $\%\left(95 \% \mathrm{CI}^{*}\right)$ & $\mathrm{P}^{\dagger}$ & $\mathrm{n}$ & $\%\left(95 \% \mathrm{CI}^{*}\right)$ & $\mathrm{P}^{\dagger}$ & $\mathrm{n}$ & $\%\left(95 \% \mathrm{CI}^{*}\right)$ \\
\hline \multicolumn{9}{|l|}{ AT NEAR } \\
\hline \multicolumn{9}{|l|}{ Age 6} \\
\hline European Caucasian & 119 & $11.1(8.8-13.4)$ & & 592 & $55.3(50.9-59.8)$ & & 359 & $33.6(29.1-38.0)$ \\
\hline East Asian & 8 & $2.7(1.1-4.4)$ & 0.001 & 200 & $68.5(63.6-73.4)$ & 0.01 & 84 & $28.8(24.3-33.2)$ \\
\hline \multicolumn{9}{|l|}{ Age 12} \\
\hline European Caucasian & 166 & $12.2(10.5-13.8)$ & & 651 & $47.7(40.7-54.6)$ & & 549 & $40.2(33.0-47.4)$ \\
\hline East Asian & 17 & $5.0(3.5-6.6)$ & 0.04 & 221 & $65.6(56.9-74.3)$ & $<0.0001$ & 99 & $29.4(20.9-37.9)$ \\
\hline \multicolumn{9}{|l|}{ AT DISTANCE } \\
\hline \multicolumn{9}{|l|}{ Age 6} \\
\hline European Caucasian & 16 & $1.5(0.7-2.3)$ & & 131 & $12.4(9.4-15.3)$ & & 913 & $86.1(83.1-89.0)$ \\
\hline East Asian & 1 & $0.4(0.0-0.9)$ & 0.2 & 55 & $19.6(14.1-25.0)$ & 0.003 & 225 & $80.1(74.6-85.6)$ \\
\hline \multicolumn{9}{|l|}{ Age 12} \\
\hline European Caucasian & 21 & $1.6(1.0-2.1)$ & & 74 & $5.5(3.0-7.9)$ & & 1263 & $93.0(90.3-95.7)$ \\
\hline East Asian & 2 & $0.6(0.0-1.4)$ & 0.2 & 46 & $13.8(7.2-20.3)$ & $<0.0001$ & 286 & $85.6(78.6-92.6)$ \\
\hline
\end{tabular}

*Confidence intervals adjusted for cluster sampling

$\dagger p$ values compare the likelihood of esophoria or exophoria rather than orthophoria when comparing children of East Asian ethnicity versus children of European Caucasian ethnicity. For esophoria at distance, numbers were too small for a meaningful statistical comparison. 


\section{The Impact of Habitual Glasses on the Measure of Heterophoria}

There were 35 children aged 6 years and 300 children aged 12 years who wore glasses habitually. Of those who had a small heterophoria (<10PD) (30 aged 6, 269 aged 12), only a small proportion had a substantial change ( $>4 \mathrm{PD})$ in heterophoria size with their glasses (6.7\% age 6 and $10.4 \%$ age 12 ). However, for those who had a large heterophoria ( $\geq 10 \mathrm{PD})(5$ aged 6, 31 aged 12) this proportion was much higher (2 aged 6 [40\%], 13 aged 12 [41.9\%]). In most cases, the change with glasses diminished the size of the heterophoria (85\% age 6 and age 12). However, seven children developed a significantly larger heterophoria (>4PD) when wearing their glasses. In all cases the glasses worn were not appropriate for the direction of their prevalent heterophoria, three become more exophoric when wearing their hyperopic prescription, and four became more esophoric when wearing their myopic prescription.

\section{Heterophoria and Heterotropia}

Figure 3 compares the proportions of heterophoria with heterotropia for the two age groups. 62 children were found to have heterotropia on cover test examination. While esophoria was uncommon for both age groups, esotropia was the predominant heterotropia, particularly for constant heterotropias. For intermittent heterotropias, the proportion of esotropia was lower (6 years $35 \%, 12$ years $25.7 \%$ ), and more closely resembled the proportion of esophoria for both near (6 years $13.6 \%, 12$ years 16.6\%) and distance (6 years $7 \%$ and 12 years $14.6 \%$ ).

However, in absolute numbers, the number of children with detectable heterophoria was much higher than the number of those with heterotropia (Figure 3).

\section{DISCUSSION}

Consistent with other studies, we found that orthophoria was the main phoric state at distance fixation ${ }^{2358}$ and the distribution of heterophoria size was leptokurtic. ${ }^{391920}$ In our population the distribution was skewed towards exophoria in both the 6 and 12 year-old children. This was true for both major ethnic groups in the sample - European Caucasian and East Asian. This tight distribution of heterophoria at distance around orthophoria suggests an active biological process of orthophorisation, as previously postulated. ${ }^{319}$ This is likely to result from the eyes being aligned for distance viewing of parallel light rays using tonic vergence ${ }^{1}$.

While many studies of similarly aged children have reported that orthophoria is the most frequent state for near fixation, ${ }^{2} 8$ we found that exophoria predominated. After stratification, this finding persisted in the European Caucasian and East Asian populations. However, the children of East Asian ethnicity had significantly more exophoria and less esophoria than European Caucasian children, even after adjustment for refractive error. This parallels the high prevalence of predominantly intermittent exotropia in East Asian populations. ${ }^{21}$ Wider IPD has been attributed to East Asian populations, ${ }^{22}$ and it has been suggested that this may be linked to a higher prevalence of exophoria. ${ }^{23}$ However, in our population there was no statistical difference in IPD measures between European Caucasian and East Asian children. There must be another mechanism, not yet identified, contributing to more exophoria amongst East Asian children.

Heterophoria measured without wearing glasses was associated with refractive error. Esophoria was associated with hyperopia for both age groups, and this parallels the well known association between esotropia and hyperopia and may be due to the need for greater 
accommodative efforts to overcome hyperopia. Acting in concert with convergence, this tendency impacts on ocular alignment, causing an esodeviation. ${ }^{13}$ Exophoria was associated with myopia, but only in the 12 year age group. This association may be due to a reduced demand for accommodation. Again, it parallels the association between intermittent exotropia, particularly convergence weakness type ${ }^{21}$ and myopia. While lack of accommodative effort may not in itself cause the ocular divergence, it may allow both eyes to assume a physiological position of rest, that is, slightly divergent. The orthophoric state may require slight amounts of accommodative convergence to maintain a parallel position of the eyes. ${ }^{1}$

While they are very small in number, those with myopia and near esophoria are important clinically, because it has been reported that progressive additional lenses can significantly reduce myopic progression in this group. ${ }^{24}$ This may help to reduce the development of pathological myopia, especially since this group is more prone to increased progression. ${ }^{25}$

Clinical experience in the area of intermittent exotropia shows that it may be preceded by a large exophoria which has decompensated and there is the expectation that intermittent esotropia may be preceded by an esophoria. In order to determine if decompensation of preexisting heterophoria is the major developmental pathway for the appearance of some heterotropias, particularly intermittent heterotropias, then longitudinal studies of heterophoria and incident heterotropia in children will be needed to establish this link. 


\section{ACKNOWLEDGMENTS}

We thank the parents and children who participated in this study, and the principals and teachers who helped to facilitate it. We also thank the many health professionals who assisted in data collection, and the teams involved in data entry.

Competing interests: None declared.

Funding: Funding for the Sydney Myopia Study was provided by a grant from the Australian National Medical \& Health Research Council (ID 253732) and the Vision Cooperative Research Centre. I. Morgan's contribution was supported by a grant from the Australian Research Council (COE561903). J. Leone holds a National Health and Medical Research Council Biomedical (Dora Lush) Postgraduate Research Scholarship (Scholarship ID 457173). Neither sponsor participated in the design or conduct of the study, collection, analysis or interpretation of the data, or in the preparation, review, or approval of the manuscript.

\section{FIGURE LEGENDS}

Figure 1 Percentage of different types of prevalent heterophoria (without glasses) in children aged 6 and 12 years, at near and at distance. The only significant differences, when comparing percentages between the two age groups occurred only at distance fixation for orthophoria ( $\mathrm{p}=0.01)$, and for exophoria $(\mathrm{p}=0.007)$.

Figure 2 Bar graphs demonstrating the distribution of prevalent heterophoria (measured at near and distance fixation) in PD (prism dioptres) for all children aged 6 and 12 years (negative values indicate exophoria and positive values indicate esophoria).

Figure 3 Proportions of horizontal heterophoria in comparison to proportions of intermittent, constant and all heterotropia in children aged 6 and 12 years. 


\section{REFERENCES}

1 Duke-Elder SS, Wybar K. System of Ophthalmology: Ocular Motility and Strabismus. London: Henry Kimpton 1973:515-62.

2 Walline JJ, Mutti DO, Zadnik K, Jones LA. Development of phoria in children. Optom Vis Sci 1998;75:605-10.

3 Letourneau JE, Giroux R. Nongaussian distribution curve of heterophorias among children. Optometry \& Vision Science 1991;68:132-7.

4 Junghans B, Kiely PM, Crewther DP, Crewther SG. Referral rates for a functional vision screening among a large cosmopolitan sample of Australian children. Ophthalmic Physiol Opt 2002;22:10-25.

5 Macfarlane DJ, Fitzgerald WJ, Stark DJ. The prevalence of ocular disorders in 1000 Queensland primary schoolchildren. Aust N Z J Ophthalmol 1987;15:161-74.

6 Gupta M, Gupta Y. A survey on refractive error and strabismus among children in a school at Aligarh. Indian J Public Health 2000;44:90-3.

7 Chen AH, O'Leary DJ, Howell ER. Near visual function in young children. Part I: Near point of convergence. Part II: Amplitude of accommodation. Part III: Near heterophoria. Ophthalmic Physiol Opt 2000;20:185-98.

8 Lam SR, LaRoche GR, De Becker I, Macpherson H. The range and variability of ophthalmological parameters in normal children aged $41 / 2$ to $51 / 2$ years.[see comment]. J Pediatr Ophthalmol Strabismus 1996;33:251-6.

9 Jackson TW, Goss DA. Variation and correlation of standard clinical phoropter tests of phorias, vergence ranges, and relative accommodation in a sample of school-age children. Journal of the American Optometric Association 1991;62:540-7.

10 Saladin JJ, Sheedy JE. Population study of fixation disparity, heterophoria, and vergence. Am J Optom Physiol Opt 1978;55:744-50.

11 Gupta NC, Narang RK, Khurana AK, Parmar IP, Ahluwalia BK. Exophoria and refractive errors-evaluation of 250 cases. Indian J Ophthalmol 1987;35:204-6.

12 Anson AM, Davis H. Diagnosis and Management of Ocular Motility Disorders. Third ed. London: Blackwell Science, 2001:312-27.

13 Lyle TK, Wybar KC. Lyle and Jackson's Practical orthoptics in the treatment of squint (and other anomalies of binocular vision). 5th ed. London: Lewis, 1967:42025.

14 Ojaimi E, Rose KA, Smith W, Morgan IG, Martin FJ, Mitchell P. Methods for a population-based study of myopia and other eye conditions in school children: the Sydney Myopia Study. Ophthalmic Epidemiol 2005;12:59-69.

15 Rainey BB, Schroeder TL, Goss DA, Grosvenor TP. Inter-examiner repeatability of heterophoria tests. Optom Vis Sci 1998;75:719-26.

16 North R, Henson DB. Adaptation to lens-induced heterophorias. Am J Optom Physiol Opt 1985;62:774-80.

17 Robaei D, Rose KA, Kifley A, Cosstick M, Ip JM, Mitchell P. Factors associated with childhood strabismus: findings from a population-based study. Ophthalmology 2006;113:1146-53.

18 Robaei D, Kifley A, Mitchell P. Factors associated with a previous diagnosis of strabismus in a population-based sample of 12-year-old Australian children. American Journal of Ophthalmology 2006;142:1085-8.

19 Dowley D. The orthophorization of heterophoria. Ophthalmic Physiol Opt 1987;7:169-74.

20 Dowley D. Heterophoria. Optom Vis Sci 1990;67:456-60. 
21 Chia A, Roy L, Seenyen L. Comitant horizontal strabismus: an Asian perspective. $\mathrm{Br}$ J Ophthalmol 2007;91:1337-40.

22 Wu KH, Tsai FJ, Li TC, Tsai CH, Peng CT, Wang TR. Normal values of inner canthal distance, interpupillary distance and palpebral fissure length in normal Chinese children in Taiwan. Acta Paediatr Taiwan 2000;41:22-7.

23 Ditmars DL. Relationship between refractive error, phoria, and interpupillary distance in 500 patients. Journal of the American Optometric Association 1966;37:361-3.

24 Gwiazda JE, Hyman L, Norton TT, Hussein ME, Marsh-Tootle W, Manny R, et al. Accommodation and related risk factors associated with myopia progression and their interaction with treatment in COMET children. Invest Ophthalmol Vis Sci 2004; 45:2143-51.

25 Goss DA. Variables related to the rate of childhood myopia progression. Optom Vis Sci 1990;67:631-6. 
Figure 1 Percentage of different types of prevalent heterophoria (without glasses) in children aged 6 and 12 years, at near and at distance. The only significant differences, when comparing percentages between the two age groups occurred only at distance fixation for orthophoria $(\mathrm{p}=0.01)$, and for exophoria $(\mathrm{p}=0.007)$.

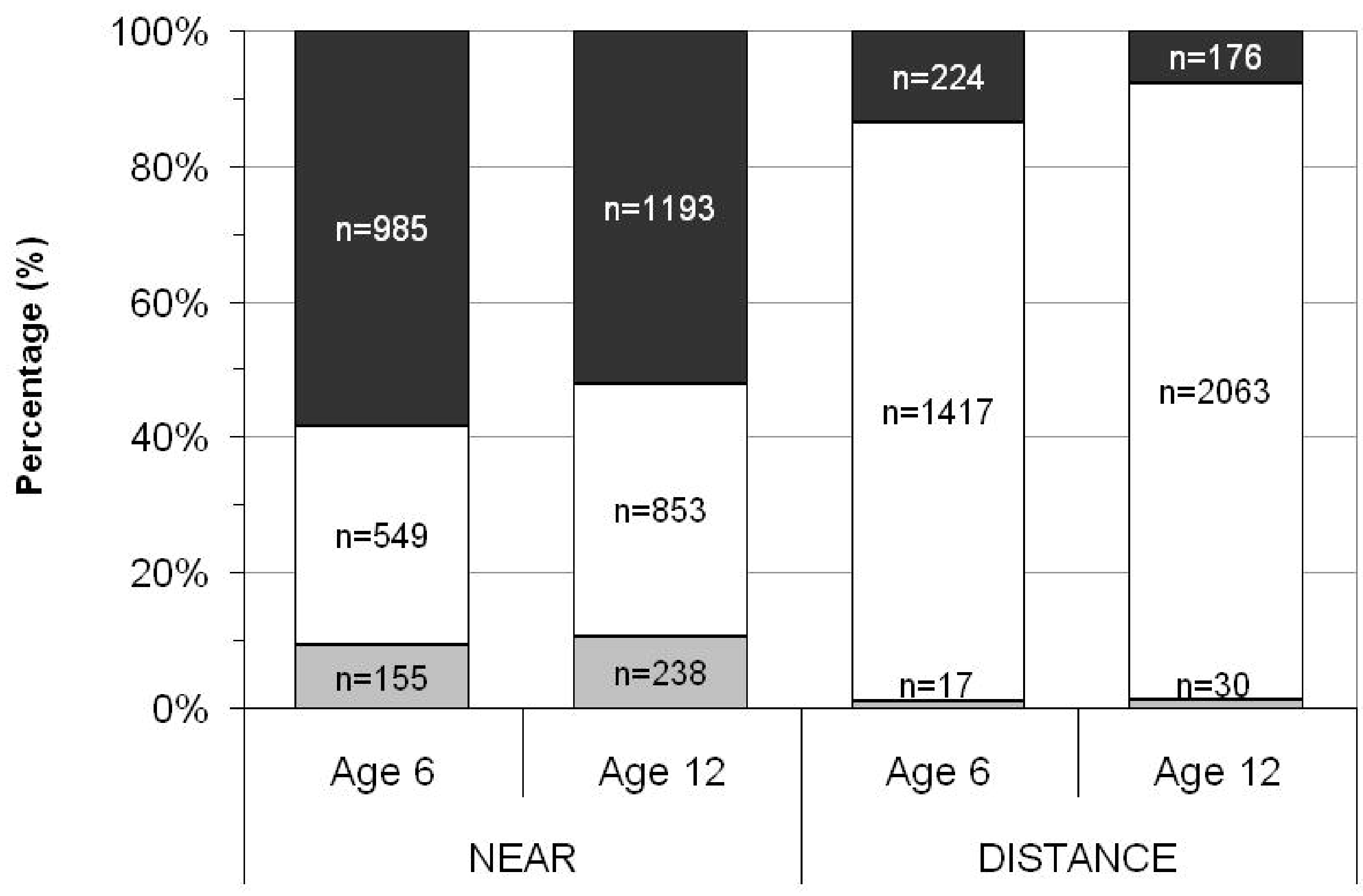

$\square$ Esophoria $\square$ Orthophoria $\square$ Exophoria 
Figure 2 Bar graphs demonstrating the distribution of prevalent heterophoria (measured at near and distance fixation) in PD (prism dioptres) for all children aged 6 and 12 years (negative values indicate exophoria and positive values indicate esophoria).
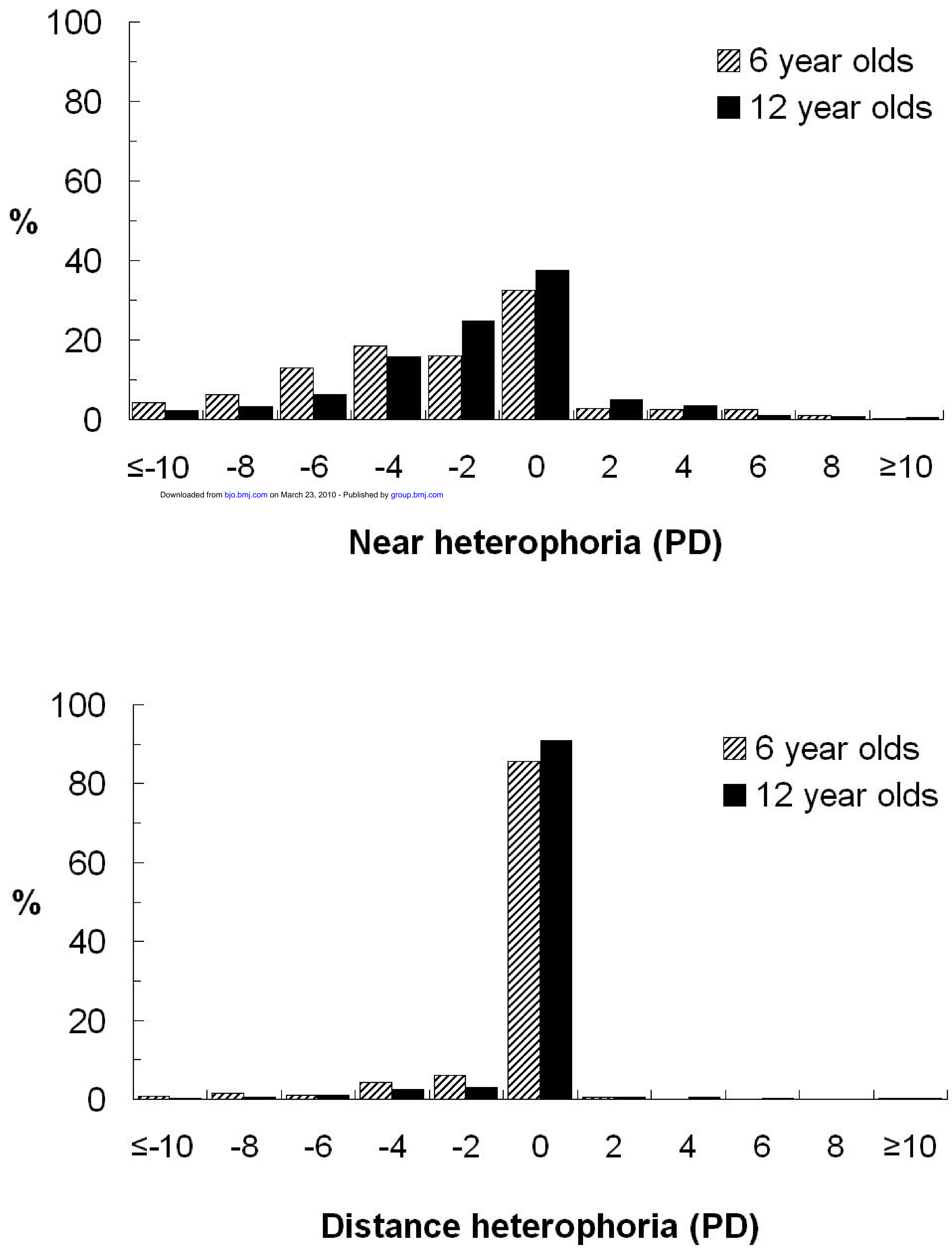
Figure 3 Proportions of horizontal heterophoria in comparison to proportions of intermittent, constant and all heterotropia in children aged 6 and 12 years.

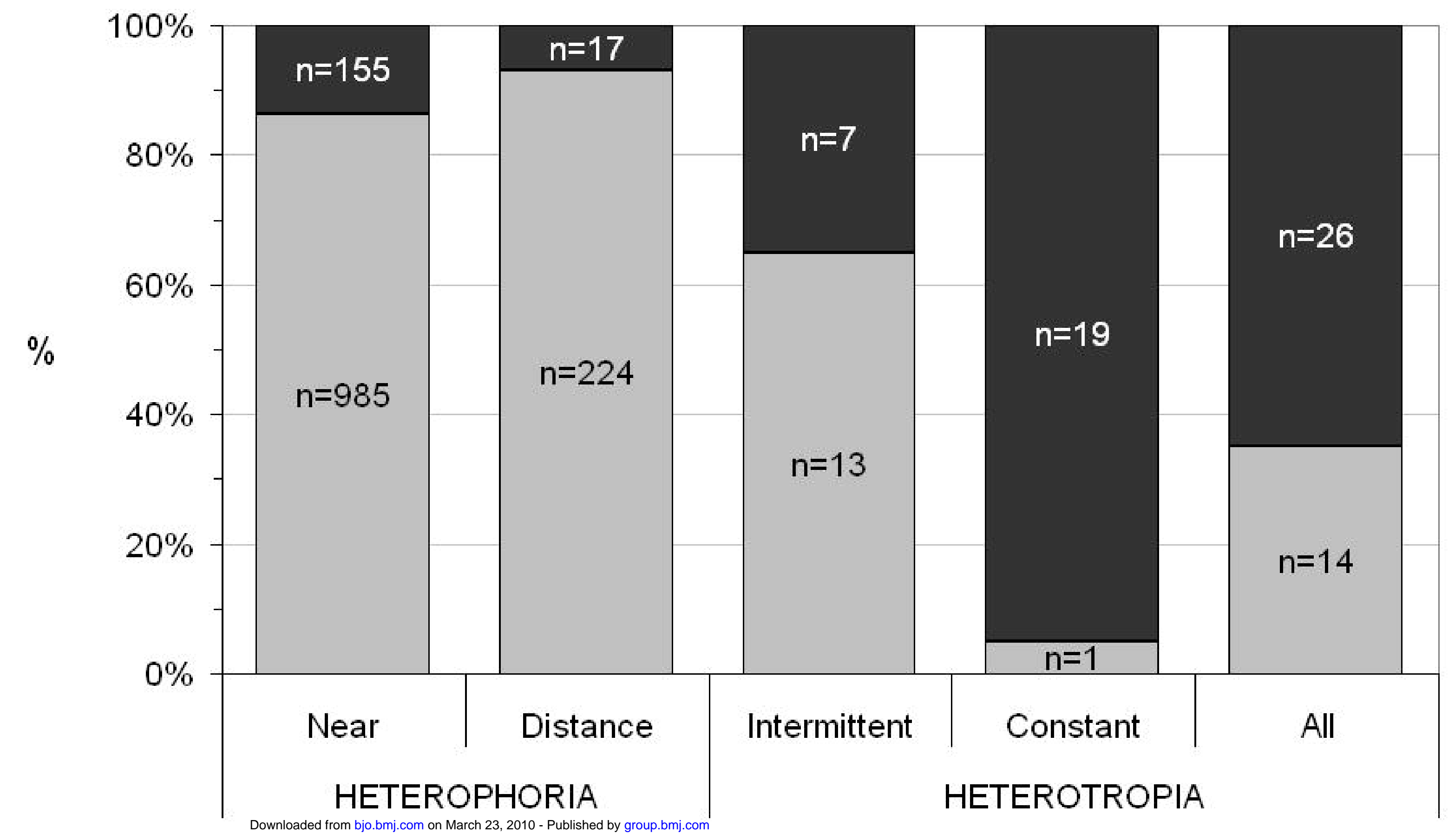

6 year old children $\square$ Exo $\square$ Eso

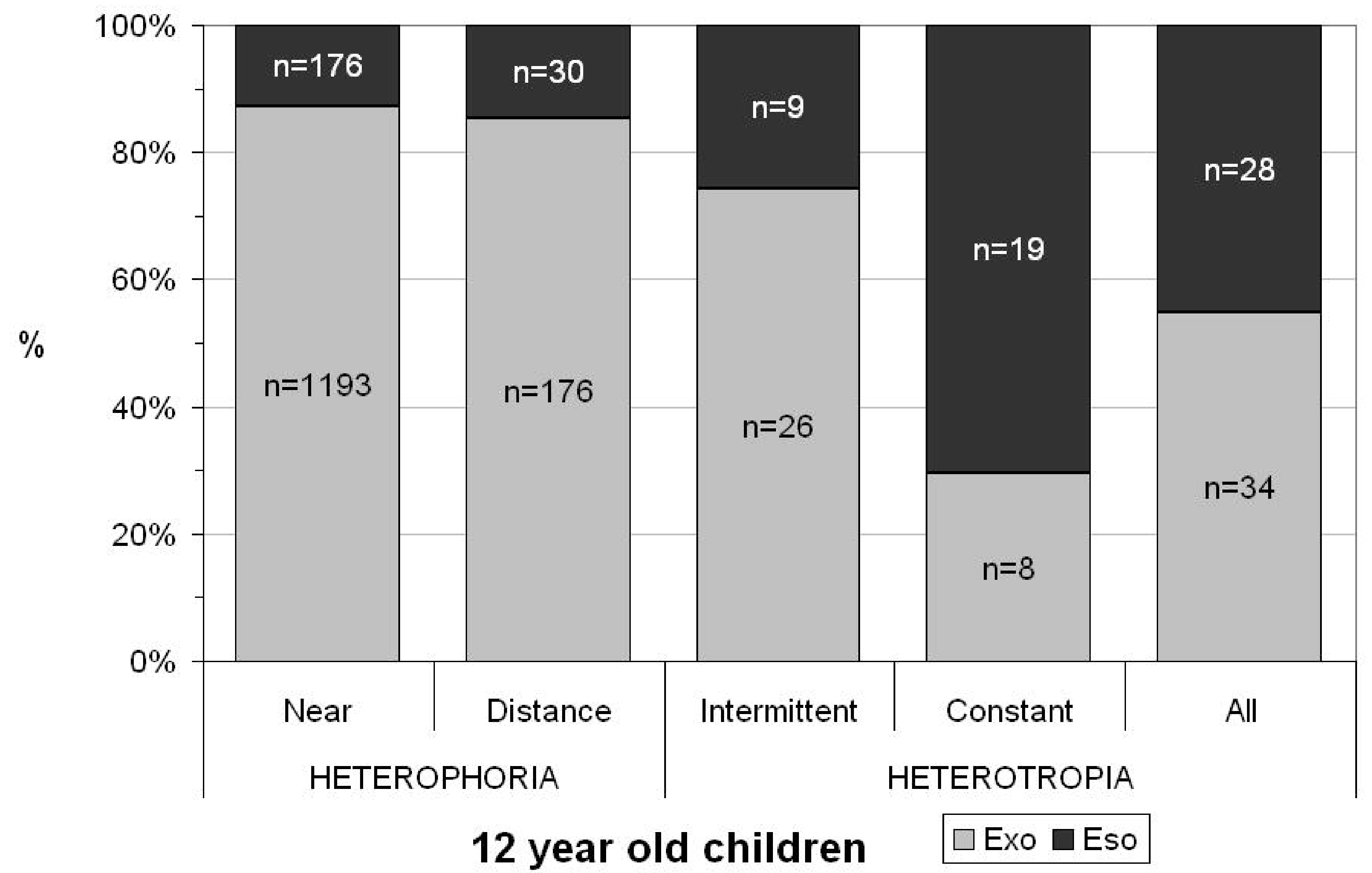

